\title{
Expecting Many
}

\section{Tim Fernando and Hans Kamp \\ University of Stuttgart}

\begin{abstract}
The determiner many is analyzed relative to a notion of expectation that is introduced into contexts employed in the theory of generalized quantifiers. Issues concerning intensionality, vagueness, ambiguity and context change are considered along the way.
\end{abstract}

\section{Introduction}

The semantics of the determiner many can be specified within generalized quantifier theory as roughly

$$
\operatorname{many}(A, B) \quad \text { iff } \quad|A \cap B|>n
$$

for some suitable lower bound $n$ on the cardinality $|A \cap B|$ of the set of $A$ 's that are $B$ 's. What is "rough" about (i) is $n$, an early proposal for which (due to Barwise and Cooper [3]) is

$$
n=\max \left(n^{\prime}, k \cdot|A|\right)
$$

for some number $n^{\prime}$ and fraction $k$ (between 0 and 1). Motivated largely by Milsark's weak/strong-classification of determiners (according to which only weak determiners can occur in there-insertion sentences). Partee [11] argues for a decoupling of $n^{\prime}$ and $k \cdot|A|$. yielding two different readings of many: a weak cardinal reading. given by (i). with $n$ supplied by the context: and a strong proportional reading

$$
\operatorname{many}(A . B) \quad \text { iff } \quad|A \cap B|>k \cdot|A|
$$

where this time the context contributes $k$. Lnder the assumption that a choice of context fixes the parameters $n$ and $k$ independently of the arguments $A$ and $B$ of many. readings (i) and (ii) can then be differentiated through various semantic properties. An example is symmetry: the conditions many $(A, B)$ and $\operatorname{many}(B . A)$ are evidently equivalent under (i) but not under (ii).

But what do such judgments amount to. given the underlying premiss that $n$ and $k$ are determined by context, irrespective of the arguments $A$ and $B$ of many? The question is somewhat delicate. as (i) or (ii) can only be defended by abstracting away the ragueness in many through a highly idealized notion of context that picks out a precise value for $n$ or $k$. Vagueness aside. however. would it not be useful to have a notion of context where either (la) or (lb) could be uttered truthfully. even if the talks had an identical audience (numbering fewer than a hundred)? 
a. Many people came to our talk.

b. Not many people came to Chomsky's talk.

Perhaps more crucially, there is the matter of logical consequence, characterized customarily by universal quantification over (fixed) contexts

$\psi$ is a logical consequence of $\varphi$ iff for every context $c$, if $\varphi$ is true at $c$ then so is $\psi$.

With a view to shedding light on these points, the present paper investigates cases in which some notion of norm or expectation determines just how many is many. We do not claim that all uses of many can be explained along these lines (a question taken up at some length in $\S 3.1$ below). Even so we believe that it is instructive to inquire into how $n$ and $k$ might, in certain cases, arise.

\subsection{The fixed context assumption and extensionality}

Let us begin with a remark from Barwise and Cooper [3] about generalized quantifier theory, which brings out a number of issues that will concern us.

One of the simplifying assumptions often made in the model theory is that one has a fixed context which determines the meaning of the basic expressions. We can think of this context as providing an interpretation for non-logical determiners ... In this paper we shall assume throughout that there is a rich context held fixed that determines the precise meaning for basic expressions, even those like "most", "many" and "few". We refer to this as the fixed context assumption. ... The fixed context assumption is our way of finessing the vagueness of non-logical determiners. We think that a theory of vagueness like that given by Kamp [1975] for other kinds of basic expressions could be superimposed on our theory. ${ }^{4}$ We do not do this here. to keep things manageable.

We will turn to hamp [6] and footnote 4 shortly, but for now let us note that (presumably "to keep things manageable") Barwise and Cooper [3] focus on determiners $Q$. for which the truth conditions of $Q x\left(\varphi, \iota^{\circ}\right)$ is given by a relation between the $x$-extensions $\{x: \varphi\}$ and $\{x: \varphi \cdot\}$ of $\varphi$ and $\varphi$. Such a scheme would appear inadequate for many. however. as illustrated by the aforementioned pair (1). and even earlier by the pair (2) from heenan and Stavi [9] (which argues for non-extensionality at the first argument of many), and (3) from \$4.4.4 of Kamp and Reyle [8] (which argues for non-extensionality at many's second argument).

(2) a. Many lawyers attended the meeting this year.

b. Many doctors attended the meeting this year.

a. Many houses in $\mathrm{X}$ burned down last year. 
b. Many houses in $\mathrm{X}$ were insured against fire last year.

In all these examples, the reasoning is the same; evaluate the pair many $(A, B)$ and many $\left(A^{\prime}, B^{\prime}\right)$ under the assumption that argument $A$ is co-extensional with $A^{\prime}$, and $B$ with $B^{\prime}$, but assign $\operatorname{many}(A, B)$ and $\operatorname{many}\left(A^{\prime}, B^{\prime}\right)$ different truth values on the basis of different expectations for $A \cap B$ and $A^{\prime} \cap B^{\prime}$.

In reply to these arguments, Partee [11] minimizes the non-extensionality of many, pointing out that "the only element of value judgment that may have to enter into the interpretation of simple many and few may be in determining a suitable value for $n$ or $k$ in the given context; but each such meaning, once $n$ or $k$ is fixed, is clearly extensional." Sweeping non-extensionality under the rug of context, she proceeds to compare the situation with past attempts "to establish whether relative adjectives like tall and expensive are intensional (like good) or, as Kamp (1975) suggested and Siegel (1976) convincingly argued, vague context-dependent extensional adjectives." While we are a little unclear which passage in Kamp [6] lends itself to this interpretation, ${ }^{1}$ the relevance of vagueness to many is obvious enough; as Partee observes,

In normal usage it seems that we do not expect the context to narrow down the choice of $n$ to a single precise value, but only to narrow it down enough to be communicatively useful.

Now, if many is, as Partee contends, "clearly extensional" "once $n$ or $k$ is fixed," then why dwell on the non-extensionality of many, when that nonextensionality can be confined to parameters $n$ and $k$ that are indisputably vague? Should we expect a precise story about how $n$ or $k$ get determined, when they, in fact. do not get determined precisely? Without a clear conception of vagueness, the matter is bound to remain vague.

\subsection{Vagueness and context-dependence}

The analysis of vagueness in hamp [6] is put very concisely in footnote 4 in Barwise and Cooper [3].

Kamp`s proposal is basically to evaluate a sentence with respect to a class of models. rather than a single model.

Although there is a bit more to Kamp [6]. this suffices to make the point that Claim 1. One need not worry about losing ragueness by positing models that resolve it away: ragueness can be recovered by constructing a context from a family of such models (being careful to distinguish the notion of a context from that of a model).

Kamp [6] act ually analyzes vagueness more directly in terms of a partial model. and passes to completions of it (corresponding to the aforementioned "family of such models") in order to define a probability function on sets of these. A function $\mathcal{C}$ is assumed that narrows truth gaps when fed a context, although not much is said about just what a context is, let alone where the function $\mathcal{C}$ comes from. 


\subsection{Partee's adjectival and determiner readings}

Returning to the word many, let us record Partee [11]'s analysis, before presenting our own. The cardinality reading (i) is exemplified by assigning (4) the discourse representation structure (DRS)

\begin{tabular}{|c|}
\hline $\mathrm{X}$ \\
\hline Many $(\mathrm{X})$ \\
Students $(\mathrm{X})$ \\
Arr-today $(\mathrm{X})$ \\
\hline
\end{tabular}

where $\mathrm{X}$ ranges over plural objects. ${ }^{2}$

$$
\text { Many students arrived today. }
$$

Line (iii) treats many as an adjective, reducing many $A$ 's $B$ to there are many $A \cap B$ 's, according to what Keenan and Stavi [9] call an existential reading. By contrast, under the proportional reading (ii), (4) is represented as the duplex condition

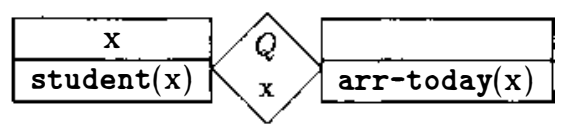

where $Q$ is many.

\section{Intensionalizing many}

Interpretations (i) and (ii) can be expressed, with the sets $A$ and $B$ replaced by formulas $\varphi$ and $\psi$, as

$$
\text { Many } x(\varphi, \psi) \quad \text { iff } \bigvee_{n \geq 1}\left(\left(\exists_{\geq n} x\right)(\varphi \wedge \psi) \wedge n \text {-is-many } x_{x}(\varphi, \psi)\right)
$$

where $\exists_{\geq n} x$ abbreviates "there are at least $n x{ }^{\prime \prime}$ " and $n$-is-many $y_{x}\left(\varphi \cdot \iota^{\prime}\right)$ intuitively expresses that " $n$ is many. for $\varphi$ - $x$ 's satisfying $2 \cdot$." The first conjunct $(\exists \geq n)(\varphi \wedge i)$ in the right hand side of $(v)$ is a straightforward existential claim. the verification of which requires only the extensions of $\varphi$ and $\psi^{\prime}$ (which are, in turn. treated in a symmetric fashion). The thorny conjunct is the second; it is here where lines (i) and (ii) diverge. Under the existential reading associated with (iii). the two arguments ( $\varphi$ and $i$ ) of $n$-is-many $x_{x}$ can be combined by conjunction. reducing our specification of many to a unary predicate $n$-IS-MANY ${ }_{x}$ (applied to $\uparrow \wedge \iota^{\circ}$ ) describing many as an adjective

$$
n \text {-IS-MANY } Y_{x}(\varphi \wedge \imath) \quad \text { iff } n \text {-is-many }{ }_{x}(\varphi, \psi) .
$$

On the other hand. for the determiner reading (ii) of many, we will need both argument places of $n$-is-many $y_{x}$. 


\subsection{Treating many as a unary predicate}

The adjective many is analyzed in (iii) not only as extensional but also as intersective. While this may do relative to a notion of context that fixes the bound $n$ in (i) independently of the arguments of many, the matter becomes quite different if the arguments are allowed to contribute to the calculation of the bound. These contributions must be encoded into the meanings the context assigns to the arguments and supplies when evaluating the utterance.

Claim 2. The context at which a sentence is uttered does not alone determine $n$ in (i); $n$ depends also on the arguments of many, which cannot be interpreted simply by their extensions.

Claim 2 is crucial to our analysis, which proceeds from

Claim 3. $n C$ 's are many when $|C|$ could well have been $<n$.

More concretely, we offer a formalization of "could well have been" by probabilities. But what should the probabilities be defined on? Should the variable $x$ used up in making the factual assertion "there are $\geq n x$ 's" be reintroduced again for calculating probabilities? This approach is blocked by, for instance, the Keenan and Stavi [9] example (2), which requires different measures for lawyers and doctors, even when they happen to be co-extensional. That is. Claim 2 essentially yields the corollary

Claim 4. Defining probabilities on objects is inadequate to capture expectations behind many.

The basic intuition about what a norm is in the cases which concern us should make clear in which direction we have to look: for alternative situations. or possible worlds. ${ }^{3}$ Accordingly, let us formalize Claim 3 in terms of a probability function $p$ on sets of worlds $(u, \ldots)$ by the equivalence

$$
\begin{aligned}
n-I S-\operatorname{MANY}_{x}(\chi) & \text { iff } \quad \text { it is probable that }\left(\exists_{<n} x\right) \Lambda^{*} \\
& \text { iff } \quad p\left(\left\{u:|\chi|_{x, u^{\prime}}<n\right\}\right)>c
\end{aligned}
$$

where $|\backslash|_{x, u}$ is defined to be $\mid\left\{x: \backslash\right.$ in $\left.u^{\prime}\right\} \mid$. For now. let us assume only that $p$ and $c$ are fixed by the model (independently of $\backslash$ ). and relate our analysis of $(v)$ to $(\mathrm{i})$ :

$$
\begin{aligned}
& \text { Many } x\left(\varphi^{\prime} \cdot \iota^{\prime}\right) \quad \text { iff } \bigvee_{n \geq 1}\left(\left(\exists_{\geq n} x\right)\left(\vartheta \wedge \iota^{\prime}\right) \wedge p\left(\left\{u^{\prime}:\left.\left.\right|_{\varphi} \wedge \iota^{\cdot}\right|_{r . u}<n\right\}\right)>()\right. \\
& \text { iff } \mid=\wedge{ }_{r} \geq n_{* \wedge \omega}
\end{aligned}
$$

where by definition. $|\backslash|_{s}=|\{x: \backslash\}|-$ assuming when no world is specified that evaluation is carried out at the actual world $\cdots$ and $n_{\chi \cdot x}= \begin{cases}\min \left\{n: p\left(\left\{u^{\prime}:|\backslash|_{x \cdot u}<n\right\}\right)>c\right\} & \text { if } \exists n p\left(\left\{u^{\prime}:|\backslash|_{r . u^{\prime}}<n\right\}\right)>c \\ x & \text { otherwise. }\end{cases}$

As noted by C. Condoravdi. we lose the empirically problematic ent ailments (5) and (6) predicted in Partee [11]. under which many is (on its cardinality reading) persistent and monotonic. 
There were many beach front houses that were flooded last year; thus, there were many houses that were flooded last year.

There were many students that came to the talk and asked questions; thus, there were many students that came to the talk.

Monotonicity and persistence break down because if either argument, $\varphi$ or $\psi$, in Many $x(\varphi, \psi)$ is weakened, the probability $p\left(\left\{w:|\varphi \wedge \psi|_{x, w}<n\right\}\right)$ could well fall below $c$, raising $n_{\varphi \wedge \psi, x}$. (The failure of these properties is unaffected by a strengthening of $\chi \supset \chi^{\prime}$ to $\square\left(\chi \supset \chi^{\prime}\right)$.)

On the positive side, a property that we may wish to meet is illustrated by (7), assuming for simplicity that there were at least two such houses.

$$
\text { Many houses in X burned down last year; in fact. all houses }
$$
in $\mathrm{X}$ burned down last year.

An account of the felicity of the use of in fact above requires a different (noncardinal) reading of many.

\subsection{Treating many as a binary relation}

Turning to the duplex analysis (iv), note that the discourse markers introduced by the restrictor remain available within the nuclear scope. In cases of intensionality, the contextual update may consist also of narrowing the collection of possible worlds on which probabilities (and $n$-is-many $\left.{ }_{r}\left(\varphi^{*}, \iota^{\prime}\right)\right)$ are defined. Recall that the conditional probability $p\left(X \mid Y^{*}\right)$ of $X^{\prime}$ gicen $Y^{\prime}$. defined as $\frac{p(X \cap Y)}{p(Y)}$ provided $p(Y) \neq 0$ (undefined. otherwise). is designed to adjust the probabilities $p$ to a domain obtained by intersection with $Y$.

Claim 5. Under a construal of many as an intensional quantifier. the restrictor may change the probabilities within its nuclear scope by conditionalization.

Asymmetry may then arise from (local) context change

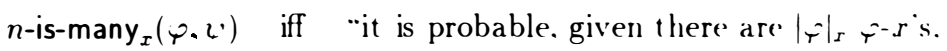

$$
\begin{aligned}
& \text { that } \exists_{<n} x\left(\varphi \wedge \iota^{\prime}\right) "
\end{aligned}
$$

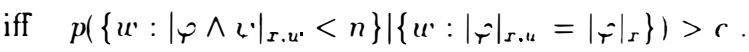

Note that all $\varphi-x$ 's count the same when relativizing to the condition $\left.\left.\right|_{T}\right|_{\text {r.u }}=$ $|\varphi|_{x}$. A minor emendation is suggested by certain (admittedly) marginal cases like (8). in which there is a logical connection between the arguments of many.
a. Many $A$ 's are $A$.
b. Many $A$ s are not $A$. 
To validate sentences of the form (8a), let us add the disjunct " $|A \cap B|$ could not have been $>n$ " (taking both $<n$ and $>n$ into account), so that

$$
\begin{aligned}
& n \text {-is-many } x_{x}(\varphi, \psi) \quad \text { iff } \quad p\left(\left\{w:|\varphi \wedge \psi|_{x, w}<n\right\} \mid W_{\varphi, x}\right)>c \text { or } \\
& p\left(\left\{w:|\varphi \wedge \psi|_{x, w}>n\right\} \mid W_{\varphi, x}\right)=0
\end{aligned}
$$

where $W_{\varphi, x}$ is an abbreviation for the set $\left\{w:|\varphi|_{x, u^{\prime}}=|\varphi|_{x}\right\}$. The additional disjunct would, for the case $n=0$, make absurd sentences of the form $(8 \mathrm{~b})$ true - but fortunately (v) skips over $n=0 .^{4}$

Note that we have derived an asymmetric reading from a symmetric one via conditionalization, without making reference to the fraction $k$ in line (ii). One candidate for $k$ is the relative frequency $\frac{|B|}{|V|}$ of $B$ (where $V$ is the set of all objects) considered in Westerståhl [14]. However, instantiating (ii) with this value yields the symmetric reading

$$
\operatorname{many}(A, B) \quad \text { iff } \quad|A \cap B|>\frac{|B|}{|V|} \cdot|A| .
$$

The idea that some notion of frequency is related to the proportional reading carries, nonetheless, some plausibility. Commenting on the proportional reading, Partee [11] writes

One subtlety that deserves further investigation is that when the restrictor does involve a very open-ended set, "proportion" ... becomes ill-defined, and some extended sense of "frequency" may be needed, including an atemporal sense conceptualized in terms of an imagined survey of the given domain. Proportion proper may then be viewable as a special case of frequency.

It is instructive at this point to return to our discussion in $\S 2.1$ of probabilities and (non-)extensionality. Notions of frequency underly a conception of probabilities defined on (sets of) objects (in, for example, a first-order model). as opposed to subjective notions of belief which lead to a definition of probabilities on (sets of) possible worlds. Now, in Partee s "imagined survey", it is. according to Claim 4 (in $§ 2.1$ ), the sampling situations (completed into worlds) on which probabilities should be defined. Thus, rather than taking the conditional probability $p(B \mid A)$ on (sets) of objects. let us introduce for every property $A$. a generic constant $a_{A}$, and calculate on worlds

$$
p_{A, B}=p\left(\left\{w^{\prime}: \llbracket a_{A^{A}} \rrbracket_{u^{\prime}} \in \llbracket B \rrbracket_{u^{\prime}}\right\} \mid\left\{w^{\prime}: \llbracket a_{A} \rrbracket_{u^{\prime}} \in \llbracket \cdot A \rrbracket_{u^{\prime}}\right\}\right) .
$$

the point being to instantiate (ii) with $k=a \cdot p_{A . B}$ for some coefficient $a$ (chosen. like $c$. independently of $A$ and $B$ )

$$
\operatorname{many}(A . B) \text { iff }|A \cap B|>\alpha \cdot p_{A . B} \cdot|A|
$$

(where $|A|$ is the cardinality of the extension of $A$ at the actual world, and similarly for $|A \cap B|)$. But now how does this proportional reading relate to 
the one given by line (vii) above? It is certainly not our intention here to argue that (vii) and (viii) represent different readings of many. On the other hand, there is no denying that for a fixed model, line (vii) may well specify an interpretation of many different from that under (viii). We conjecture, however, that discrepancies at individual models evaporate once we turn our attention to what is valid at all models satisfying certain natural conditions. These conditions concern the ingredients $p, c$ and $\alpha$ employed above, about which we have so far been deliberately vague.

\subsection{Some loose ends}

Turning to the numbers $c$ and $\alpha$ that quantify just how well is "well" in Claim 3 (i.e., $n C$ 's are many when $|C|$ could well have been $<n$ ), let us first observe that the passage from $\S 2.1$ to $\S 2.2$ via conditionalization can be strengthened (i.e., reversed), provided the same values for $c$ and $a$ are used in analyzing the cardinal and proportional readings. More precisely, observe that we might derive a cardinal reading from a proportional reading by stipulating

$$
n \text {-IS-MANY }{ }_{x}(\chi) \quad \text { iff } n \text {-is-many }{ }_{x}(x=x, \chi) \text {. }
$$

Claim 6. The unary predicate many (in §2.1) can be derived from the binary relation many (in $\S 2.2$ ) by putting the common noun predicate in the nuclear scope.

Claim 6 asserts that the cardinal reading from line (vi) (in §2.1) can be ob-

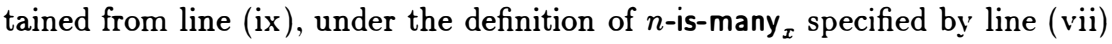
or (viii) (in §2.2). Again, such equivalences may not hold at any fixed model. but only between certain models where the cardinality $|\mathrm{V}|$ of the universal set $V$ is sufficiently larger than $|C|$ (and $|A|$ and $|B|$ ) so that the restriction to worlds with a fixed cardinality has no effect on our expectations about $C^{\prime}$ (or $A$ or $B)$.

A consequence of Claim 6 of potentially wider linguistic interest is the suggestion that an existential reading of Many $x(\varphi, \dot{\psi})$ moves $\varphi$ over to be conjoined with $\psi-$ rather than $\psi$ over to $\varphi$. That is.

$$
\text { there are many } C \text { 's iff many }(\text { I.C) . }
$$

instead of the equivalence

$$
\text { there are many } C \text { 's iff } \operatorname{many}(C . V)
$$

adopted (for instance) in Partee [11]. ${ }^{5}$

Next. let us consider what the values of $c$ and $a$ are. Why not simply set $c=\frac{1}{2}$ and $a=1$ ? It is useful here to compare the determiner many with most.

(9) a. Many A's $B$. Many $A$ 's not $B$. 

b. Most $A$ 's $B$. Most $A$ 's not $B$.
c. There are many $A$ 's.

(9a) is satisfiable, whereas $(9 \mathrm{~b})$ is not. But cardinalities and probabilities measure sets in a similar way insofar as $|A-B|=|A|-|A \cap B|$ and $p(X-Y)=$ $p(X)-p(X \cap Y)$ (switching here from $A$ and $B$ to $X$ and $Y$, in order to remind the reader that we define probabilities not on sets $A$ and $B$ of objects but on sets $X$ and $Y$ of possible worlds). It follows that (9a) cannot be satisfiable under a proportional reading (vii) where $c=\frac{1}{2}$ or (viii) where $\alpha=1$. Under the cardinal reading (vi), (9a) would imply (9c). But do we always want to infer $(9 c)$, given $(9 a)$ ?

With a bit more work, we might argue that for any positive integer $m$, it would be wrong to require of all models that $c$ and $\alpha$ be greater than $\frac{1}{m}$. Consider an election with say, $2^{m}$ candidates, $c_{1}, c_{2} \ldots, c_{2^{m}}$.

Many of the women voted (only) for candidate $c_{i}$.

For $m+1$ (of the possible $2^{m}$ ) sentences in (10) to be simultaneously satisfiable under a proportional reading (suggested by the partitive construction), we need a threshold $c$ (or $\alpha$ ) below $\frac{1}{m}$.

Although this argument loses its plausibility as $m$ approaches infinity, it would seem prudent to think twice about fixing $c$ or $\alpha$ once and for all. But if $c$ and $\alpha$ are not fixed, how do they vary? If $c$ or $\alpha$ were to depend on either argument $A$ or $B$ of $\operatorname{many}(A, B)$, then we would have complicated many with intensions. only to shove the problem of $n$ 's and $k$ s dependence on $A$ and $B$ (in (i) and (ii)) over to $c$ and $\alpha$. We must instead resort to the assumption that variations in values of $c$ and $a$ can be accounted for by variations in models. Furthermore. such values must, at any model, be constant.

Intensionality Hypothesis. There are enough possible worlds differentiating distinct properties so that $c$ and a need not depend on the arguments of many, but can. for any given model. stay fixed.

The Intensionality Hypothesis is very possibly the most problematic (and interesting) point of the present work. Can any two judgments $n$-is-many ${ }_{x}\left(\varphi, \iota^{\circ}\right)$ and $n$-is-many ${ }_{x}\left(\varphi^{\prime} \cdot \iota^{\prime}\right)$ be glued together by a common bound $c$ or $a$ ? Or are probabilities defined on total worlds overkill, in which case would we be better advised to base our estimation of when $n x-x$ s are many some other way? What matters ultimately, we believe, is not so much the interpretation of many at any particular model, but rather the validities and rules predicted by quant ifying over a suitable collection of these models (with different $p$ s, $c$ s and $\alpha$ s). These validities and rules may turn out to be so trivial as to admit a simpler non-probabilistic semantic interpretation, pushing the elimination of numbers from probabilities in Segerberg [13] further. Unfortunately, we must leave the development of such technical points to another paper. 


\section{Discussion}

The fundamental intuition about many on which $\S 2$ rests is expressed by Claim $3, n C$ 's are many when $|C|$ could well have been $<n$. It is natural to interpret the phrase "could well have been" relative to what we know about $C$, which, comprising only a small part of the information encoded in the actual world, leads to so-called subjective probabilities, requiring (in turn) an intensional framework. ${ }^{6}$

\subsection{Expecting many readings}

While it is plausible to assume that expectations must ultimately be grounded in the actual world, exactly how that comes about is undoubtedly an extremely complicated story. We need not spin such a tale, however, in order to give an extensional analysis of many that goes beyond simply assuming that context spits out $n$. A step in this direction is to follow Lappin [10] and add a comparison set argument to many, turning it into a ternary predicate ambiguous between

$$
\operatorname{many}_{1}(A, B, C) \quad \text { iff } \quad|A \cap B|>|A \cap C|
$$

and

$$
\operatorname{many}_{2}(A, B, C) \text { iff } \quad|A \cap B|>|B \cap C|,
$$

suitable for sentences such as (11).

Many lawyers are criminals, as compared to doctors.

But what do we do with sentences that do not spell out a comparison set C? A framework eliciting missing arguments from context is the alternative semantics for focus of Rooth [12]. The idea is that an utterance of a sentence specifies an expression in focus (written between brackets $[\cdot]_{F}$ ), that is (somehow) assigned a set $\mathcal{A}$ of alternatives, relative to which the truth of the sentence is evaluated. For example, Lappin [10]'s interpretations of (11) amount to an alternative semantics for $(12 \mathrm{~b})$ and $(12 \mathrm{c})$, with the alternative set $\mathcal{A}=\{$ doctors $\}$.
a. There are many lawyers who are criminals.
b. There are many lawyers who are $[\text { criminals }]_{F}$.
c. There are many [lawyers $]_{F}$ who are criminals.

The matter becomes more complicated in case the set $\mathcal{A}$ of alternatives has more than one element. This possibility is considered carefully in Babko [2]'s analysis of cardinal many as a focus-sensitive quantifier, for which a bit of notation comes in handy. Let $\chi$ be a formula with a free variable $x$, such as lawyer $(x) \wedge \operatorname{criminal}(x)$. and suppose $e$ is a subexpression in $\chi$ that is 
in focus. Given an element $a$ in the alternative set $\mathcal{A}$, let $\chi_{e}^{a}$ be the formula obtained by replacing $e$ in $\chi$ by $a .^{7}$ A simple proposal for when $n \chi$-x s can be deemed many (relative to $\mathcal{A}$ ) is

$$
n \chi-x \text { 's are many (relative to } \mathcal{A}) \quad \text { iff } \quad(\forall a \in \mathcal{A})\left|\left\{x: \chi_{e}^{a}\right\}\right|<n .
$$

Babko dismisses $(\mathrm{x})$ and any characterization that depends only on the cardinality of $\mathcal{A}$ and the number of $a$ 's in $\mathcal{A}$ for which $\left|\left\{x: \chi_{e}^{a}\right\}\right|<n$. Instead, she calculates a lower bound for $n$, using (in an essential way) the numbers $\left|\left\{x: \chi_{e}^{a}\right\}\right|$ defined by alternatives $a$ in $\mathcal{A}$. And even then, she acknowledges "potential problems with such definitions."

Now, the relevance of this little digression to our analysis $(\S 2)$ is that if we introduce into the formula $\chi$ a variable $w$ for the world (taking intensions to extensions) on which to apply $[\cdot]_{F}$ (setting $e$ to $w$ ), then our probabilistic proposals can be viewed as special cases of the alternative scheme above, where $\mathcal{A}$ is some set of possible worlds (restricted in the case of $\S 2.2$ to worlds meeting some condition imposed by the restrictor $\varphi$ in Many $x(\varphi, \psi))$. Instead of $(\mathrm{x})$, we have

$$
\begin{aligned}
n \chi-x \text { 's are many iff } \quad\left\{a \in \mathcal{A}:\left|\left\{x: \chi_{w}^{a}\right\}\right|<n\right\} \text { is } \\
\\
\text { a "sizable" subset of } \mathcal{A}
\end{aligned}
$$

for some notion of "sizable" subset defined by a probability function $p$ and number $c$ (or $\alpha){ }^{8} \quad \mathrm{By}$ weakening the universal quantifier in $(\mathrm{x})$. (xi) reduces the demands on the context to specify $\mathcal{A}$ as precisely as it must under $(\mathrm{x})$.

Within the alternative semantics of focus, our account is distinguished by the application of $[\cdot]_{F}$ to an implicit intensional argument. This bit of intensionality might be avoided by applying []$_{F}$ instead to an explicit argument. Babko states that

... the alternative semantics of focus is all that is needed in order to adequately account for the context-dependency of 'many' and 'few'.

This claim is somewhat unsettling to the extent that (a) the alternative semantics of focus stops at some presumed boundary between semantics and pragmatics. ${ }^{9}$ and (b) the contention that many is extensional is defended by erecting such borders. To our knowledge. Babko makes no such defense. She does consider a different analysis of many based on focus due to Herburger [5]. under which "the cardinal/proportional distinction does not. coincide with Milsark's strong/weak distinction." and goes on to write

A basic argument for the cardinal/proportional ambiguity of 'few' and 'many'. however. comes from Milsark's observations about the weak character of the cardinal 'many' and the strong nature of the proportional one. The present analysis preserves this insight, since all weak interpretations of 'many' and ' $f\left(w^{\prime}\right.$ ' are considered to be cardinal and focus-affected. whereas all proportional readings are strong. 
But now consider the apparent asymmetry in certain there-insertion sentences such as $(13 \mathrm{ab})$.
a. There are many lawyers who are criminals.
b. There are many criminals who are lawyers.
c. Many lawyers are criminals.
d. Many criminals are lawyers.

Even if an appeal is allowed to some topic/focus structure to transform (13a,b) to what amount to proportional readings of $(13 c, d)$, it is difficult to resist the nagging sense that there is something wrong in insisting on the semantic principle that cardinal equals weak and proportional equals strong, only to fall back on pragmatics to account for (pragmatically innocent) counter-examples of the form There are many $A$ 's who are $B$ 's.

Turning our attention back to the case where $\mathcal{A}$ is a set of possible worlds, let us acknowledge that even in this case, general questions of (a) contextdependence and of (b) where $\mathcal{A}$ comes from remain. Item (a) is taken up very briefly in the next section; (b) in the section after that.

\subsection{The fixed context assumption, extensionality, vagueness and ambiguity reconsidered}

Barwise and Cooper [3]'s "fixed context assumption" stating that "there is a rich context held fixed that determines the precise meaning for" (among other expressions) many is challenged above not only by the claim that extensions are not quite "rich" enough (Claim 2), but also by our appeal. for an asymmetric reading. to context change, in the form of conditionalization of probabilities. Since this conditionalization is local to the scope, one might want to discount this context change, and portray it as an ordinary case of context-dependence (whatever that amounts to). As we will see shortly, however, there could well be other situations where conditionalization is not local. At any rate, the proposal above to (re-)analyze there are many $C$ s as many $(V, C)$ (where $V$ can be read: "vacuous") is meant to suggest that this context-dependence consists of some form of domain restriction (relating the adjectival and determiner readings of many).

Describing context-dependence can (needless to say) be a tricky matter, even when the task is simply to decide when it is that context resolves vagueness. and when it is that it resolves ambiguity. As a first approximation, one might say that vagueness has to do with choosing the model against which to evaluate a logical form: by contrast, ambiguity concerns the choice of logical form (to evaluate). Stated this way, notions of vagueness and ambiguity presuppose a fixed conception of logical form and model. That is, the demarcation between vagueness and ambiguity varies with different notions of logical form and model - which can get quite confusing when it is not clear just what 
can be put into the logical form. For example, adding the annotation $[\cdot]_{F}$ from Rooth [12] leads to (a) ambiguity because it can be attached to different expressions in a sentence, and to (b) vagueness insofar as there is a choice in the alternative set to interpret a fixed attachment. As for the probabilistic analyses presented above, the Intensionality Hypothesis in $\S 2.3$ says that the choice of $c$ and $\alpha$ is up to the model, not the logical form (as $c$ and $\alpha$ are independent of the arguments of many). In that respect, the indeterminacy in $c$ and $\alpha$ (as well as $p$ ) leads to vagueness, not ambiguity.

That is not to say, of course, that ambiguity does not arise when using expectations. In addition to the cardinal/proportional ambiguity, there are various expectations to keep track of, leading to different readings. Consider sentence (14), which would seem paradoxical under a reading of many as more than (could have been) expected.

As expected, many students arrived today.

Such a contradiction is easy enough to resolve along the following lines: the expectation underlying many above might concern arrivals on days other than. or in addition to, today; the expectation referred to in as expected pertains specifically to today. More generally, all kinds of probabilities might be involved, conditionalized on, or applied to, any number of things.

\subsection{Playing with expectations}

We close with a puzzle that begins by asking for lower bounds on many for each line of (15).

a. Many people came to the colloquium.

b. Chomsky spoke. Many people came to the colloquium.

c. Tim spoke. Many people came to the colloquium.

Chances are it is easier to pick out a number for (15b) than for (15a). and that the number for (15c) is far lower than (15b). due to the acceptability of (16).

a. If Chomsky speaks, many people will come to the colloquium.

b. If Tim speaks, not many people will come to the colloquium.

Now. the puzzle is that the set $\mathcal{A}$ of possible worlds on which $n$-is-many ${ }_{x}$ is evaluated seems to change in (15b) and (15c) but not in (16a) or (16b). ${ }^{10}$ Evidently. $\mathcal{A}$ is not immune to the first sentences in (1.5b) and (15c). but escapes update by the antecedents in (16a) and (16b).

We are not sure exactly how to account for this discrepancy. One clue is that. in contrast to (15). many is used in (16) not to report a particular incident. but rather to express expectations, such as (1i).

Many birds fly. 
(Curiously, the expectations raised by (16) would seem to work towards making (15b) and (15c) equally likely - perhaps to increase their information content.) Just what the existential import of (16) or (17) is falls outside the scope of the present work, although it is easy enough to assign (16) and (17) a semantics where the existential quantification in line $(\mathrm{v})$ of $\S 2$ has no counterpart. Alas, if we have stuck to line $(\mathrm{v})$, it is because we have tried not to stray too far away from uses of many considered in Partee [11], where "meaning, once $n$ or $k$ is fixed, is clearly extensional." And even there we have failed.

\section{fernando@ims.uni-stuttgart.de hans@ims.uni-stuttgart.de}

\section{Endnotes}

*Many thanks to Cleo Condoravdi for helpful discussions.

${ }^{1}$ In any case, neither of us currently believes that the extensionality claim is correct.

${ }^{2}$ As argued in Kamp and Reyle [8], NPs beginning with many do not introduce set discourse referents directly, but only via Abstraction, as in

$$
\begin{array}{|c|}
\hline \mathrm{X} \\
\hline \mathrm{X}=\sum \mathrm{x} .(\text { student }(\mathrm{x}) \wedge \operatorname{arr}-\operatorname{today}(\mathrm{x})) \\
\operatorname{Many}(\mathrm{X})
\end{array}
$$

This is to make sure that the many phrase receives a distributive interpretation. For the discussions in this paper, the difference is of no importance.

${ }^{3}$ The term "possible world" may be somewhat misleading in this context, for there are many cases where we think of the alternative situations in terms of which the norm is determined as alternative situations in the actual world. See in this connection $\S 3.1$.

${ }^{4}$ It is possible that because we perceive that the norm strategy clearly makes no sense for a sentence like $(8 \mathrm{a})$, we are provoked into another interpretation strategy that is more appropriate to it. Adding the alternative possibility as another disjunct to the truth clause may not be the optimal way of accounting for this. We will leave this problem to a companion paper which addresses the logic which our semantics generates.

${ }^{5}$ As pointed out by $C$. Condoravdi, the proposal to re-analyze there are many $C$ s as many $(V, C)$, rather than many $(C, V)$, can be made without bringing in the issue of intensionality. contrary to the presentation above. Setting intensionality aside, one might ask whether the manner in which the arguments of a determiner are instantiated reflects more generally the weak/strong ambiguity of quantifiers $Q$. In particular, does not the vacuous restriction in $Q(V, C)$ support (or otherwise relate to) the contention (e.g., in Zucchi [15]) that weak 
NPs are not subject to Heim [4]'s Descriptive Content Condition (inasmuch as the only presupposition $Q(V, C)$ adds is that the universe $V$ be non-empty)? Note that the interpretation of the adjective many (under a weak existential reading) makes essential use only of the nuclear scope. By contrast, the restrictor plays an indispensable role for strong quantifiers such as most (e.g.: Kamp [7]) and, under a proportional reading, many.

${ }^{6}$ The word "subjective" in subjective probabilities suggests that the collection of possible worlds represents the doxastic alternatives entertained by an idealized logically omniscient agent, and that if we are to go beyond this idealization, intensionality must give way to intentionality.

${ }^{7}$ Interpreting many relative to extensional alternatives $\chi_{e}^{a}$ is faithful to Claim 3 only insofar as $\chi_{e}^{a}$ resemble $\chi$, so that the switch $a / e$ could be viewed intensionally as moving to a most "similar world" modulo $a / e$. The question is would the word "expection" still be interesting after its meaning is stretched far enough to cover any such alternative semantics for many?

${ }^{8}$ Our probabilistic approach constitutes a qualitative alternative to calculating the expectation $E(v)$ of a random variable $v$

$$
E(v)=\sum n \cdot p(v=n) .
$$

The latter (quantitative) approach comes closer to Babko`s aforementioned lower bounds for $n$.

${ }^{9} \mathrm{By}$ contrast, let us cite Asher [1] as a daring work that pushes the analysis of context-dependence beyond such borders.

${ }^{10}$ We are indebted here to $\mathrm{R}$. Schwarzchild for asking how $\mathcal{A}$ relates to the notion of common ground (in say. Heim [4]).

\section{References}

[1] Nicholas Asher. From discourse macro-structure to micro-structure and back. Manuscript. distributed at a workshop on context dependency in Prague. 1995.

[2] Olga Babko-Malaya. How many is many? Draft. Rutgers Iniversity. February 199.5.

[3] Jon Barwise and Robin Cooper. Generalized quantifiers and natural language. Linguistics and Philosophy. 4. 1981.

[4] Irene Heim. The semantics of definite and indefinite nom phrases. Dissertation. Eniversity of Massachusetts. Amherst. 1982.

[5] Elena Herburger. Focus and the LF of NP Quantification. In Proc. S.1LT 3. 1993.

[6] Hans Kamp. Two theories about adjectives. In E. Keenan. editor. Formal Semantics of . Vatural Language. Cambridge ('niversity Press. 1975).

[i] Hans hamp. What a linguist might want from a logic of Most and other generalized quantifiers. Manuscript. 1996. 
[8] H. Kamp and U. Reyle. From Discourse to Logic. Kluwer Academic Publishers, Dordrecht, 1993.

[9] E. Keenan and J. Stavi. A semantic characterization of natural language determiners. Linguistics and Philosophy, 9(3), 1986.

[10] Shalom Lappin. Many as a two-place determiner function. Manuscript, 199 ?

[11] Barbara H. Partee. Many quantifiers. In J. Powers and K. de Jong, editors, Proceedings of the Fifth Eastern States Conference on Linguistics (ESCOL). The Ohio State University, Columbus, 1988.

[12] Mats Rooth. Association with focus. Dissertation, University of Massachusetts, Amherst, 1985.

[13] Krister Segerberg. Qualitative probability in a modal setting. In J.E. Fenstad, editor, Second Scandinavian Logic Symposium, pages 341-352. North-Holland, 1971.

[14] Dag Westerståhl. Logical constants in quantifier languages. Linguistics and Philosophy, 8, 1985.

[15] Alessandro Zucchi. The ingredients of definiteness and the definiteness effect. Natural Language Semantics, 3, 1995. 